\title{
Subgingival Curettage
}

National Cancer Institute

\section{Source}

National Cancer Institute. Subgingival Curettage. NCI Thesaurus. Code C63713.

Removal of degenerated and necrotic epithelium and underlying connective tissue of a periodontal pocket in an effort to convert a chronic ulcerated wound to an acute surgical wound, thereby insuring wound healing and attachment or epithelial adhesion, and shrinkage of the marginal gingiva. 